\title{
Análisis Económico de la Infidelidad Sexual
}

Juan León Mendoza

Resumen: Mediante la formulación de un modelo de corte microeconómico se analiza el fenómeno de la infidelidad sexual de la población masculina. La infidelidad sexual es un acto racional y se efectúa bajo una lógica maximizadota de beneficios (bienestar o placer) sujeto a restricciones o costos. Si el beneficio marginal que reporta la infidelidad es mayor que el costo marginal, entonces, el individuo tenderá a incurrir en actos de infidelidad sexual hasta el punto en que el costo marginal sea igual al beneficio marginal.

Palabras claves: Análisis económico, infidelidad sexual. 


\section{Juan León Mendoza}

\section{Introducción}

Un taxista me comentaba que "casi no conocía amigos o personas que no hayan sido infieles alguna vez a sus parejas sentimentales". En una sociedad occidental relativamente machista y cada vez más liberal, los varones tienden a mantener alguna relación sexual ocasional, esporádica o estable con una mujer o mujeres diferentes a sus parejas formales

¿Porqué tiende a producirse la infidelidad sexual?. ¿Cuáles son las variables explicativas?. Con la finalidad de responder a las preguntas planteadas, formularemos un modelo teórico de corte microeconómico.

El análisis económico de la infidelidad sexual puede parecer un tema banal, morboso o irrelevante. También puede parecer una pérdida de tiempo en la medida en que existen muchos y grandes problemas económicos, los cuales el economista debe tratar de investigar, conocer y darle solución. Sin embargo, con el presente artículo queremos mostrar que las actividades de la infidelidad sexual, en esencia, no sólo tienen una racionalidad económica, sino, que la herramienta de la ciencia económica puede ser muy útil para entender el comportamiento humano en el campo sexual.

La aplicación de la teoría económica al entendimiento de la conducta humana fuera del campo económico no es una novedad. Gary Becker, se hizo acreedor al Premio Nóbel de Economía en el año 1992 justamente por haber abordado el matrimonio, el divorcio, la delincuencia, la familia, la discriminación sexual, entre otros fenómenos extraeconómicos, desde la óptica de la ciencia económica.

Sin embargo, Gary Becker no abordó específicamente el fenómeno actualmente recurrente y cada vez mucho más notorio: la infidelidad sexual. Con el presente artículo trataremos de cubrir dicho vacío además de relativizar la teoría de Freud,-quien sostuvo que el comportamiento humano estaba motivado fundamentalmente por las pulsaciones sexuales mediante la endogenización de la racionalidad sexual con la racionalidad económica, es decir, mostrando el predominio del homus economicos sobre al homus sexual.

El artículo es teórico. Crearemos una serie de conceptos y categorías económicosexuales, y mediante la formulación de un modelo microeconómico, trataremos de mostrar y explicar las razones por las cuales los individuos de sexo masculino tienden a practicar la infidelidad sexual. 


\section{Análisis económico de la infidelidad sexual}

\section{Supuestos}

- El varón que practica la infidelidad sexual es un agente racional que maximiza utilidad (satisfacción o bienestar individual) sujeto a ciertas restricciones.

- La relación sexual con la pareja formal (legal) es un bien; también la relación con una pareja informal (ilegal). Es decir, ambos tipos de actividad sexual generan beneficios a la persona que lo practica.

- La relación sexual con la pareja formal y la informal son bienes sustitutos.

- La satisfacción que genera las está sujeto al principio económico de los beneficios marginales decrecientes.

- Los varones poseen tendencias polixesuales intrínsicas.

\section{La preferencia sexual}

En principio, definimos como una persona polisexual a aquel que mantiene también relaciones sexuales con otras personas además de su pareja formal (legal). Entendemos por pareja formal o legal del varón a aquella mujer que está en situación de esposa, conviviente, novia o enamorada "oficial" (la "principal"), en tanto que la pareja informal (ilegal) es aquella que está en situación de amante (permanente o eventual) o que ocupa una menor importancia en el ranking de preferencias sexuales del individuo.

Bajo el supuesto de que el varón es un agente racional maximizador de beneficios o placer, se tiene la siguiente función de beneficios (B) a maximizar:

$$
\mathrm{B}=\mathrm{B}(\mathrm{O}, \mathrm{C})
$$

Donde: $\mathrm{O}$ representa horas de ocio y $\mathrm{C}$ bienes de consumo

Las horas de ocio se distribuyen entre el tiempo destinado a la relación sexual con la pareja formal (h.L), con la pareja informal (h.I) y las horas de descanso (D):

$$
\mathrm{O}=\text { h.L }+ \text { h.I }+\mathrm{D}
$$




\section{Juan León Mendoza}

Donde: h es la duración promedio del acto sexual, L es el número de actos sexuales con la pareja legal e, I es el número de actos sexuales con la pareja ilegal. Las horas de descanso (D) comprenden el tiempo destinado a dormir, jugar, practicar algún hobby, el aseo, ver televisión, etc., es decir, es el periodo de ocio no destinado a la actividad sexual.

La dotación de tiempo (T) que dispone la persona está dado y se distribuye entre el ocio y el trabajo $(\mathrm{N})$ :

$$
\mathrm{T}=\mathrm{O}+\mathrm{N}=\text { h.L }+ \text { h.I }+\mathrm{D}+\mathrm{N}
$$

Alternativamente, si la persona no trabaja, sino que es un estudiante, entonces, se puede reemplazar las horas destinadas al trabajo con el de estudio $(\mathrm{E})$ :

$$
\mathrm{T}=\mathrm{O}+\mathrm{E}=\mathrm{h} \cdot \mathrm{L}+\mathrm{h} \cdot \mathrm{I}+\mathrm{D}+\mathrm{E}
$$

La lógica de la ecuación (3) y (4) es de fácil entendimiento. Por ejemplo, en un día, las 24 horas que dispone la persona se agotan necesariamente entre lo que se destina al ocio y al trabajo.

Reemplazando (2) en (1) se tiene: $\quad \mathrm{B}=\mathrm{B}(\mathrm{O}=\mathrm{h} \cdot \mathrm{L}+\mathrm{h} . \mathrm{I}+\mathrm{D}, \mathrm{C})=\mathrm{B}(\mathrm{O}=\mathrm{L}+\mathrm{I}+\mathrm{D}, \mathrm{C})$

$$
\begin{aligned}
& \text { Se asume que: } \quad\left(\mathrm{B}_{\mathrm{L}}=\frac{\partial B}{\partial L}=\mathrm{BMgL}\right)>0 \\
& \mathrm{~B}_{\mathrm{LL}}<0 \\
& \mathrm{~B}_{\mathrm{I}}=\mathrm{BMgI}>0 \\
& \mathrm{~B}_{\mathrm{II}}<0
\end{aligned}
$$

$\mathrm{B}_{\mathrm{L}}>0$ y $\mathrm{B}_{\mathrm{LL}}<0$, indican que el beneficio o placer marginal de la relación sexual con la pareja legal $(\mathrm{BMgL})$ es positivo y decreciente. De la misma manera, $\mathrm{B}_{\mathrm{I}}>0$ y $\mathrm{B}_{\mathrm{II}}<0$, señalan que el beneficio marginal de la relación sexual con la pareja ilegal (BMgI) también es positivo y decreciente.

El placer que se obtiene durante la relación sexual en forma similar que cualquier bien o servicio que satisface los deseos o las preferencias del individuo está sujeto al principio económico del beneficio marginal decreciente. Manteniendo los demás variables constantes, el beneficio o la satisfacción marginal de una relación sexual disminuye en el medida en que aumenta el número de actos sexuales independientemente de que la pareja sea la legal o la ilegal. 


\section{Análisis económico de la infidelidad sexual}

Por ejemplo, dado el grado de amor o sentimiento hacia la enamorada, las primeras relacionadas sexuales efectuadas implican una gran satisfacción, pero, en la medida en que aumenta el número de relaciones sexuales, la relación sexual adicional ya no genera la intensidad de satisfacción similar a las primeras; éstas decrecen. Cuando en el tiempo, el beneficio marginal de la relación sexual tiende a llegar a cero, desaparece o se minimiza la atracción sexual por la pareja.

Sucede algo similar en las relaciones matrimoniales. Las primeras horas, días, semanas, meses o años de matrimonio, implican una relación de pareja y de sexo con altos niveles de satisfacción (beneficios marginales elevados). Sin embargo, en la medida que transcurre el tiempo, el beneficio marginal de cada relación sexual adicional necesariamente tiende a disminuir.

La tendencia decreciente de la atracción sexual que siente un varón por la pareja se confunde muchas veces con la disminución del deseo sexual. El deseo sexual es inherente a la persona, lo que disminuye es la preferencia o satisfacción marginal que se experimenta.

El principio del beneficio marginal decreciente de las relaciones sexuales con las parejas legales y las ilegales se muestra en el gráfico 1 :

\section{Gráfico 1: Beneficio marginal de la relación sexual}

BMgL

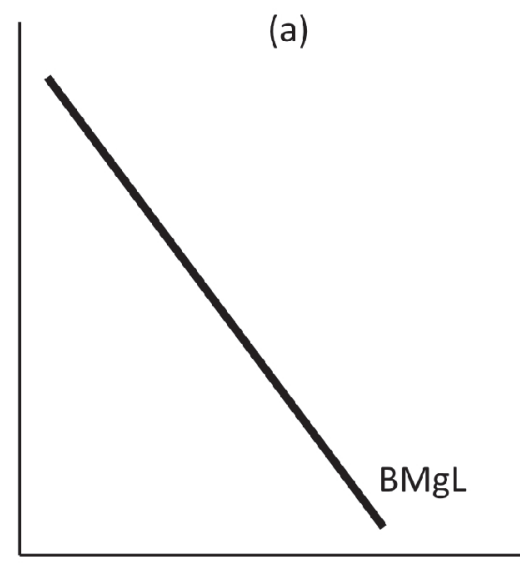

$\mathrm{BMgl}$

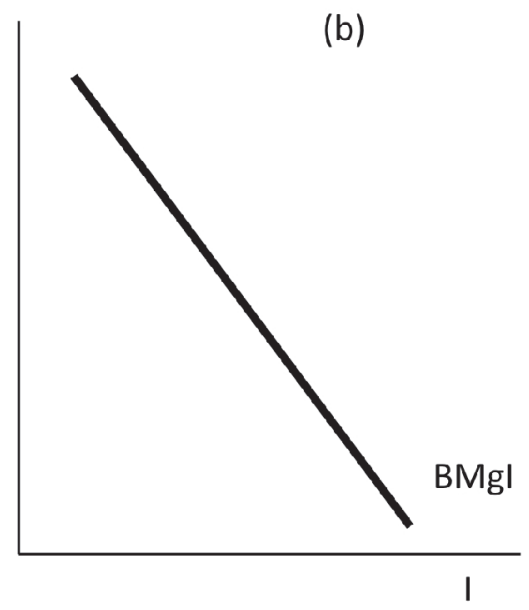


El panel (a) muestra el caso del beneficio marginal decreciente de mantener relaciones sexuales con la pareja legal y el panel (b) con la ilegal. La curva de $\mathrm{BMgL}$ con pendiente negativa muestra que, cuando aumenta el número de relaciones sexuales con la pareja legal, el beneficio marginal tiende a caer. Se tiene la misma lógica para la curva del BMgI.

El desplazamiento de la curva de $\mathrm{BMgL}$ hacia la derecha (izquierda) implica un aumento exógeno (disminución) en el beneficio marginal de mantener la relación sexual con la pareja formal. El BMgL puede caer si disminuye el grado de enamoramiento o simpatía hacia la pareja, cuando se incurre en la relación monótona y, cuando se tiene un deterioro en la apariencia personal y la presentación física de la pareja. Un aumento en el BMgL (traslado de la curva del $\mathrm{BMgL}$ hacia la derecha) puede producirse si la pareja efectúa cambios e innovaciones permanentes en su relación.

La maximización de beneficio que se alcanza cuando se mantiene relaciones sexuales con la pareja ilegal no es independiente del beneficio que se obtiene con la legal. Ambas curvas de beneficio marginal se consideran interdependientes. Cambios exógenos en la curva del $\mathrm{BMgL}$ influirá en la curva del BMgI, pero, en sentido contrario.

Por ejemplo, una disminución en el grado de enamoramiento o simpatía hacia la pareja formal desplaza la curva del $\mathrm{BMgL}$ hacia la izquierda, lo cual también repercute en el traslado de la curva del BMgl hacia la derecha. En otros términos, si el varón empieza a desilusionarse, frustrarse, desencantarse y tener conflictos recurrentes con la pareja formal, la satisfacción marginal de tener una relación sexual con ella tenderá a disminuir, por lo que el beneficio marginal de establecer una relación de pareja y de sexo con otra mujer de manera ilegal se torna atractivo y potencialmente de mayor nivel.

La lógica de la función de beneficios de la ecuación (5) se puede exponer también gráficamente en el plano del número de relaciones sexuales con la pareja ilegal (I) y la legal (L). En el citado plano, se pueden graficar un conjunto de "curvas de indiferencia sexual" (CIS) de pendiente negativa y convexa respecto al origen, los cuales reflejarían el nivel de las preferencias o deseos sexuales. El individuo maximizador deseará alcanzar o ubicarse en la CIS más alta posible, es decir, el más lejos del origen. La pendiente de la CIS sería igual a:

$$
\text { (6) } \frac{\partial I}{\partial L}=-\frac{B M g L}{B M g I}=-\mathrm{TMS}_{\mathrm{LI}}
$$




\section{Análisis económico de la infidelidad sexual}

Por el supuesto de que los beneficios marginales - tanto de la relación sexual con la pareja legal y la ilegal son decrecientes - la tasa marginal de sustitución ente I y $\mathrm{F}\left(\mathrm{TMS}_{\mathrm{LI}}\right)$ sería de signo negativo y decreciente. Sin embargo, el deseo o la preferencia sexual del varón reflejado en la CIS tiene que ser compatible con la capacidad sexual. En otros términos, el deseo de alcanzar el máximo beneficio o satisfacción en la relación sexual está sujeto a la capacidad y posibilidad sexual del individuo.

\section{La capacidad sexual}

La capacidad de efectuar las relaciones sexuales podemos representarlo en un especie de una "función de producción sexual". En tal sentido, el número de relaciones sexuales con la pareja formal depende de la capacidad física $(\mathrm{F})$, la capacidad psicológica $(\mathrm{P})$ y del grado de legalidad (GL):

$$
\mathrm{L}=\mathrm{L}(\mathrm{F}, \mathrm{P}, \mathrm{GL})
$$

El grado de legalidad tiene que ver con el grado de compromiso, aceptación y autorización familiar de la relación establecida por la pareja. Cuando mayor sea el grado de compromiso, se dice que el grado de legalidad es mayor y afecta en sentido directo el número de relaciones sexuales con la pareja legal.

La capacidad física se refiere a la resistencia o fortaleza física del individuo. La capacidad psicológica comprende a la capacidad mental para el desempeño sexual. Se asume que a mayor capacidad física y mental, el individuo podrá efectuar un mayor número de actividades sexuales, es decir, alcanzaría una mayor productividad sexual.

Dado el grado de legalidad, ee asume: $\quad \mathrm{L}_{\mathrm{F}}>0$ y $\mathrm{L}_{\mathrm{FF}}<0, \mathrm{~L}_{\mathrm{P}}>0$ y $\mathrm{L}_{\mathrm{PP}}<0$

Lo anterior indica que, el desempeño o la productividad marginal de la fortaleza física y de la fortaleza psíquica en las relaciones sexuales con la pareja formal son positivos y decrecientes. Esta productividad marginal sexual decreciente está relacionado con el principio económico de la productividad marginal decreciente de los factores de producción.

El número de relaciones sexuales con la pareja informal depende, además de la capacidad física y psicológica, de las oportunidades ilegales (OI), es decir, oportunidades sexuales con otras mujeres diferentes a la pareja legal. 
$\mathrm{I}=\mathrm{I}(\mathrm{F}, \mathrm{P}, \mathrm{OI})$

También se asumeque: $\quad \mathrm{I}_{\mathrm{F}}>0$ y $\quad \mathrm{I}_{\mathrm{FF}}<0, \mathrm{I}_{\mathrm{P}}>0$ y $\mathrm{I}_{\mathrm{PP}}<0$

Las oportunidades sexuales ilegales depende del capital facial (Kf) y del capital no humano $(\mathrm{KNH})$ :

$$
\mathrm{OI}=\mathrm{OI}(\mathrm{Kf}, \mathrm{KNH})
$$

El capital facial se refiere a la apariencia física del individuo y el capital no humano es una proxy de la riqueza económica y financiera. Se asume que, en la medida en que el individuo tienda a mostrar una mejor apariencia física según los parámetros culturales vigentes y que muestre mayores niveles de poder económico y financiero, tendrá mayores oportunidades sexuales con parejas diferentes a la pareja legal. Si un varón es considerado por las mujeres como una persona de apariencia física muy cautivante o atractivo, éste tendrá mayores pretendientes femeninas o que tendrá mayores facilidades para conquistarlos. De la misma, dado que las mujeres racionalmente tienden a preferir a personas de mayor solvencia económica y financiera, los individuos que muestren mayores niveles de riqueza tendrán también mayores facilidades para establecer nuevas relaciones informales.

Dada la dotación de la capacidad física y psíquica del varón para las relaciones sexuales, estas capacidades sexuales se distribuyen entre las parejas legales y las ilegales, estableciéndose un trade off en el nivel de producción sexual entre ambos tipos de pareja sexual.

La lógica expuesta basadas en las ecuaciones (7) y (8), se puede representar gráficamente en el plano del número de relaciones sexuales con la pareja legal (L) y la ilegal (I) mediante una curva cóncava respecto al origen y que muy bien podemos denominarlo como la "frontera de posibilidades de producción sexual" o "curva de posibilidades sexuales" (CPS).

La CPS puede desplazarse hacia el origen o alejarse de ella. Si tiende al origen, estaría mostrando una disminución en la capacidad sexual del individuo, en tanto que si se aleja, mostraría una mayor capacidad sexual. La citada curva tenderá a desplazarse de manera paralela cuando se modifica la dotación de la capacidad física y mental disponible del individuo para las relaciones sexuales. En tanto que, una mayor dotación de capital facial y el capital no humano hará rotar la CPS en el eje de I, lo cual reflejaría el hecho de que el individuo incrementa su capacidad y posibilidad de establecer un mayor número de relaciones sexuales con parejas ilegales. 
La concavidad de la CPS está relacionada a nuestro supuesto de la productividad marginal sexual decreciente de la capacidad física y psíquica del individuo, lo cual refleja a su vez la existencia del costo de oportunidad creciente en la medida en que el individuo opta por incurrir en actos de infidelidad sexual.

La decisión de mantener las relaciones sexuales con una pareja ilegal no sólo genera beneficios o satisfacciones para el individuo, sino también implica costos y dentro de los costos, el costos de oportunidad es de mucha importancia. En un sentido amplio, el costo de oportunidad está relacionado básicamente al número de relaciones sexuales que se deja de efectuar con la pareja legal, la pérdida del disfrute y desarrollo en la relación padre-hijo en el caso en que se tienen hijos, entre otros.

El costo de mantener una relación sexual con una mujer informal comprende también el valor de los bienes y recursos asignados al mismo. Además incluye, los costos que implican el riesgo de contagiarse con el virus del SIDA y el riesgo de ser descubierto la infidelidad (por la pareja legal), lo cual puede llegar inclusive a niveles de ruptura de la relación formal mantenida.

A mayor número de relaciones sexuales con la pareja ilegal, aumenta el costo total de dicha relación; por ello, dada la productividad marginal sexual decreciente y por ende el costo de oportunidad creciente plasmadas en una curva de posibilidades sexuales de pendiente negativa se genera también un costo marginal creciente.

El costo marginal creciente de establecer relaciones sexuales con la pareja ilegal (CMgI) se puede ilustrar gráficamente en el plano del número de relaciones sexuales con la ilegal (I) y el $\mathrm{CMgI}$ mediante una curva de pendiente positiva, tal como se tiene en el gráfico 2:

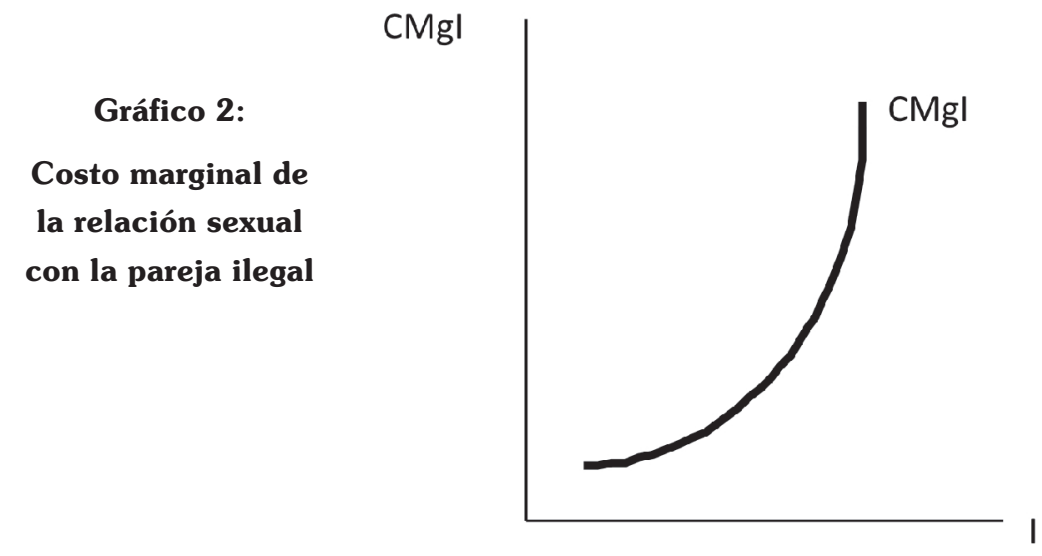




\section{Juan León Mendoza}

La curva del $\mathrm{CMgI}$ se puede trasladar hacia arriba (aumentar el costo marginal) si el individuo tiene hijos con la pareja ilegal y el riesgo en que se incurre es mayor. También puede trasladarse hacia abajo (disminuir el costo marginal) si el individuo tiene mayores oportunidades sexuales relacionadas a su mayor dotación del capital facial y, de riqueza económica y financiera.

\section{El comportamiento sexual}

La decisión del individuo de cometer actos de infidelidad sexual genera beneficios, pero también costos. El beneficio consiste básicamente en la satisfacción o placer alcanzado, en tanto que el costo está relacionado al costo de oportunidad, a los recursos que se tiene que destinar para ello y el riesgo en que se incurre. El individuo infiel, es un agente racional que maximiza beneficios pero sujeto a restricciones y costos.

En el marco del modelo formulado, la maximización de beneficios no se produce en términos totales, sino en términos marginales. Si el costo marginal de efectuar un acto de infidelidad sexual es mayor que el beneficio marginal, entonces el individuo tenderá a ser fiel a su pareja. Si el beneficio marginal es mayor que el costo marginal, el individuo racional y maximizador tenderá a optar por cometer actos de infidelidad hasta el punto en que el beneficio marginal sea igual al costo marginal. Este es el punto en que el individuo alcanza el máximo beneficio condicionado.

¿Porqué el beneficio marginal de ser infiel podría tender a ser mayor del costo marginal del mismo?. Según el modelo teórico que formulamos, dado el riesgo, ello es probable básicamente cuando: (a) disminuye el grado de enamoramiento (estimación y simpatía) hacia la pareja formal, se incurre en una relación monótona y, cuando se tiene un deterioro en la presentación y apariencia física de la pareja y, (b) en la medida en que sea mayor la dotación de capital facial y mayor la riqueza económica y financiera que posee el individuo. El argumento (a) se traduce en un aumento en el beneficio marginal de la infidelidad, en tanto que (b) en una reducción de los costos marginales. Lo manifestado podemos ilustrarlo gráficamente.

Según el gráfico 3, en una situación inicial, el costo marginal (CMgI) podría ser mayor que el beneficio marginal de establecer una relación sexual con una pareja ilegal $\left(\mathrm{BMgl}_{0}\right)$. En este caso no se tiene actos de infidelidad. Ello pertenece a la situación en que la relación con la pareja legal es excelente tal que probablemente el individuo no tiene ninguna intensión o interés de cometer actos de infidelidad. También comprende el hecho de que el costo de transacción, los costos de oportunidad y riesgo de la infidelidad, se consideran elevados. 


\section{Gráfico 3: Infidelidad sexual por incrementos en el beneficio marginal}

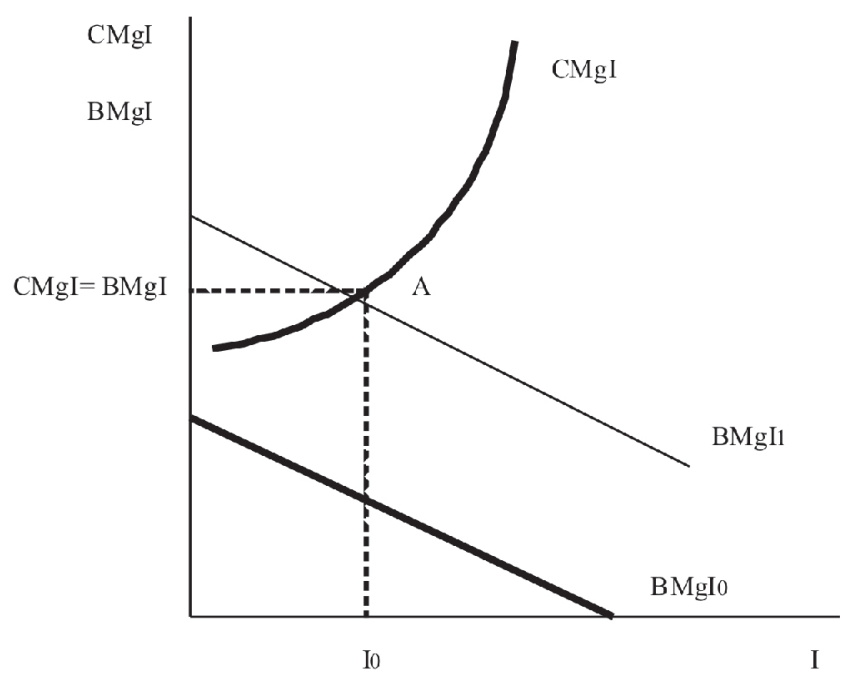

Sin embargo, dados la curva del costo marginal y el número de las relaciones sexuales informales, si disminuye el beneficio marginal de mantener relaciones sexuales con la pareja formal, entonces aumenta el beneficio marginal de ser infiel, de manera que la curva de beneficio marginal de mantener relaciones sexuales con una mujer ilegal se traslada de $\mathrm{BMgl}_{\circ}$ a $\mathrm{BMgI}_{1}$. Este hecho genera que el beneficio marginal sea mayor que el costo marginal, por lo que el individuo tenderá a practicar e incrementar el número de actos sexuales hasta $\mathrm{I}_{0}$ en el que el costo marginal es igual al beneficio marginal.

Lo expuesto en el gráfico 3 corresponde al argumento (a) ya especificado. Efectivamente, el beneficio marginal de mantener relaciones sexuales con la pareja formal disminuye en la medida en que se mantiene conflictos recurrentes, cae el grado de estimación y amor, se tiene una relación monótona y aburrida, existe un deterioro en el aspecto físico de la persona ya sea por la desatención de ella o por el paso de los años, etc., lo que tiende a incrementar el beneficio marginal de una relación sexual con una mujer ilegal, traduciéndose ello en el inicio y el aumento en la propensión marginal a la infidelidad y la práctica del mismo.

La generación de actos de infidelidad relacionados al argumento (b) se muestra en el gráfico 4. En el citado gráfico, partimos de una situación inicial en que no existe infidelidad sexual, debido a que el costo marginal es mayor que el beneficio marginal, tal que no se produce la intersección entre las curvas de $\mathrm{BMgl}$ y el $\mathrm{Cmgl}_{0}$. 
Sin embargo, dados el beneficio marginal y el número de relaciones sexuales con la pareja informal, el costo de oportunidad marginal o costo marginal de incurrir en actividades de infidelidad sexual pueden disminuir cuando aumentan las oportunidades sexuales ilegales y ello a su vez puede deberse dado el riesgo - a factores de ventaja comparativa que cuente el individuo en la dotación de capital facial y la riqueza económica y financiera. En otros términos en a medida en que el individuo sea considerado físicamente muy atractivo e "irresistible" por las mujeres, o cuando éste tenga un mayor poder económico, financiero, laboral político, etc., tenderá a disminuir el costo marginal de establecer relaciones de pareja con mujeres ilegales. Como consecuencia de ello, el costo marginal de la infidelidad será menor que el beneficio marginal, lo cual inducirá al individuo maximizador de bienestar a incurrir en actividades de infidelidad sexual hasta el punto en que el costo marginal sea igual al beneficio marginal.

En el gráfico 4, el costo marginal disminuye de $\mathrm{CMgI}_{0}$ a $\mathrm{CMgI}_{1}$, por lo que el costo marginal es menor que el beneficio marginal, lo cual induce al individuo a efectuar actividades de infidelidad sexual hasta un nivel de $1_{0}$ en la que alcanza el máximo nivel de satisfacción condicionado.

En el caso de la enfermedad relacionada al virus del sida, en tanto que este fenómeno se masifique y que por ende sea mayor el riesgo de contagiarse con el mismo por efectuar actos de infidelidad sexual, la curva de costo marginal se desplazaría hacia arriba (aumenta el costo marginal), lo que se traduciría en una reducción en el número de actos de infidelidad sexual o inclusive en la desaparición del mismo. En términos del gráfico 4, el proceso sería en el sentido inverso a lo simulado para una reducción en el costo marginal.

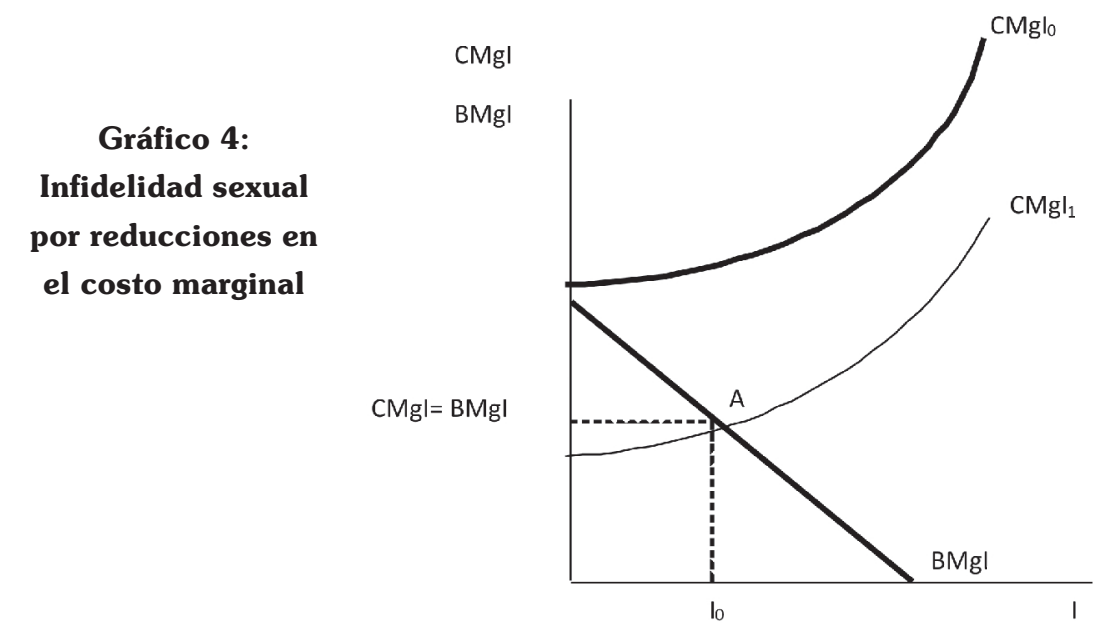




\section{Análisis económico de la infidelidad sexual}

En conclusión, según el modelo teórico formulado, el individuo que decide practicar la infidelidad sexual lo hace de manera racional y maximizadora de beneficios (satisfacciones, placeres, bienestar). La infidelidad genera beneficio y costos. La maximización de beneficios se produce en el punto en que el costo marginal de tener una relación sexual con la pareja ilegal es igual al beneficio o placer marginal que reporta ello. Si el costo marginal es mayor que el beneficio marginal y éstos no se intersectan en términos gráficos, entonces, no se genera la infidelidad. Pero, si el beneficio marginal es mayor que el costo marginal, en el marco del principio del beneficio marginal decreciente y costo marginal creciente, el individuo incurre en actividades de infidelidad sexual hasta el punto en que la satisfacción marginal que genera el último acto sexual con la pareja ilegal sea igual al costo marginal. En tal sentido, la infidelidad tenderá a producirse en la medida en que: disminuya el aprecio y amor a la pareja formal, se tenga una relación monótona, se deteriora la apariencia y presentad física de a pareja legal, cuando mayores sean a dotación del capital facial y el poder económico y financiero del individuo.

\section{Notas Referenciales}

1 Aunque actualmente tal hecho no sólo es una práctica exclusiva de los varones, sino también crecientemente de las mujeres.

${ }^{2}$ Tal como se hizo mención en la entrega del premio Nóbel: "Por haber extendido el dominio del análisis microeconómico hacia nuevos dominios del comportamiento y de las relaciones humanas, incluso más allá de los límites del mercado".

${ }^{3}$ No se tiene que ser necesariamente un infiel o un infiel experto para explicar la conducta de los que cometen actos de infidelidad sexual. En forma similar que Gary Becker (1968) que sin ser un delincuente teoriza la actividad delictiva de los delincuentes, aquí teorizamos la infidelidad sexual tratando de captar e identificar la racionalidad y las principales variables que explican el mismo. Un trabajo que aplica la economía al análisis de las decisiones sexuales, pero no de la infidelidad, se puede encontrar en Coyle (2006).

${ }^{4}$ Existen individuos para los cuales la infidelidad sexual es un mal, ello ya sea por factores religiosos, de principios, valores, cultura, coherencia con la promesa de fidelidad a la pareja formal, etc. 


\section{Juan León Mendoza}

5 En términos criollos, la pareja ilegal es la "sucursal", la "trampa" o la de "choque y fuga". Asumimos como equivalente la definición de legal con la formal y la de ilegal con la informal. Aquí no analizamos el caso de los homosexuales o de aquellos que además de contar con una pareja formal de sexo femenino tienen otra pareja informal de sexo masculino. Por otro lado, tratamos como equivalentes los conceptos de polisexo, poligamia, poliamor o poliamante.

${ }^{6}$ El término de la duración acto sexual no sólo comprende el período del coito, sino a todo el tiempo asignado en torno al mismo. Asumimos que el tiempo de duración de un acto sexual con la pareja legal es similar con el de la pareja ilegal y es igual a 1.

${ }^{7}$ Estamos asumiendo que la persona estudia (probablemente cuando sea joven) o trabaja

${ }^{8}$ Según Parkin et.al (2006). El principio del beneficio marginal decreciente indica que "cuanto más tenemos de cualquier bien o servicio, tanto más pequeño es el beneficio marginal de el” (pág. 36).

9 "Usualmente se dice que "desaparece el amor" o que disminuye el "apetito sexual" hacia la pareja. Por otro lado, si bien se ha mencionado al decrecimiento del beneficio marginal de la relación sexual en el tiempo, tal principio también es aplicable dentro de una unidad de tiempo

${ }^{10}$ Si la relación con la pareja ilegal fuera un mal, entonces las CIS tendrían pendiente positiva.

${ }^{11}$ En un sentido estrecho, definimos el costo de oportunidad como el número de relaciones sexuales que se deja de efectuar con la pareja legal por cada unidad de relaciones sexuales adicionales con la ilegal

12 A mayor nivel de la riqueza económica que posee el individuo, éste tendrá mayores posibilidades de encontrar mujeres que estén dispuestos a mantener alguna relación sentimental o sexual; la riqueza "atrae a la mujer como la miel a las moscas", "un hombre con dinero" se convierte en "más atractivo" por no decir "más rentable"- para las féminas. Como se dice usualmente, para muchas mujeres la verdadera "pepa" de un hombre no radica en la cara, sino, en su bolsillo o billetera. Sin embargo, lo manifestado no quiere decir que las mujeres venden sexo por dinero, sino que, en el contexto en que las mujeres de nuestra sociedad contemporánea todavía no gozan de las mismas oportunidades y facilidades que los varones para su desarrollo, ellas buscan y prefieren racionalmente establecer relaciones de pareja que les pueda brindar cierta seguridad, protección, crecimiento, bienestar económico y social. 
Análisis económico de la infidelidad sexual

\section{Bibliografía}

BECKER, Gary (1968). "Crime and punishment: an economic approach". The Journal of Political Economy No 76, págs. 169-217.

COYLE, Diane (2006) Sexo, drogas y economía. Editorial ITP Paraninfo, Madrid.

FREUD, Sigismund (1978) Tres ensayos de teoría sexual y otras obras. Amorrotu, Buenos Aires.

PARKIN, M., ESQUIVEL, M., y AVALOS, M. (2006) Microeconomía. Pearson Addison Wesley, México. 